\title{
On Continuous Homotopic One Layer Routing
}

\author{
Shaodi Gao*, Mark Jerrum**, Michael Kaufmann* \\ Kurt Mehlhorn*, Wolfgang Rülling*, Christoph Storb* \\ * FB 10, Universität des Saarlandes, 6600 Saarbrücken, West Germany. \\ ** Dep. of Comp. Science, University of Edinburgh, Edinburgh EH9 3JZ, Scotland.
}

\begin{abstract}
We give an $O\left(n^{3} \cdot \log n\right)$ time and $\mathbf{O}\left(\mathbf{n}^{3}\right)$ space algorithm for the continuous homotopic one layer routing problem. The main contribution is an extension of the sweep paradigm to a universal cover space of the plane.
\end{abstract}

\section{Problem Definition}

An input of the continuous homotopic one layer routing problem (CHRP) consists of a set $W=$ $\left\{w_{1}, \ldots, w_{l}\right\}$ of paths (also called wires) and a set $O \subseteq$ $\mathbf{R}^{2}$ of obstacles. A path is a continuous curve. We assume that wires do not intersect and that they are disjoint from all obstacles.

A solution to a CHRP is a set $P=\left\{p_{1}, \ldots, p_{l}\right\}$ of paths which is obtained by a homotopic shift (precise definition below) from $\mathrm{W}$ such that $U\left(p_{i}\right):=$ $\left\{x ; \operatorname{dist}\left(x, p_{i}\right)<1 / 2\right\}$ is a simply connected region of the plane, $U\left(p_{i}\right) \cap U\left(p_{j}\right)=\emptyset$ for $i \neq j$ and $U\left(p_{i}\right) \cap$ $U(O)=\emptyset \forall i$. Here dist denotes the Euclidian distance. An output to a CHRP is either a solution or the indication that no solution exists. Figure 1 gives an example of a CHRP.

Let us now give precise definitions.

Supported by DFG, SFB 124, TP B2.

Permission to copy without fee all or part of this material is granted provided that the copies are not made or distributed for direct commercial advantage, the ACM copyright notice and the title of the publication and its date appear, and notice is given that copying is by permission of the Association for Computing Machinery. To copy otherwise, or to republish, requires a fee and/or specific permission.

(C) 1988 ACM 0-89791-270-5/88/0006/0392 $\$ 1.50$
A path is a continuous function $p:[0,1] \rightarrow \mathbf{R}^{2}$. A set $W=\left\{w_{1}, \ldots, w_{l}\right\}$ of paths is collisionfree if $w_{i}(t) \neq w_{j}(s)$ for $(i, t) \neq(j, s)$ and $w_{i}(t) \notin O$ for all $i$ and $t$. So an input of a CHRP consists of a set $O$ of obstacles and a collisionfree set $W$ of paths.

We call two paths $p$ and $q$ homotopic $(p \sim q)$ with respect to a set $F \subseteq \mathbf{R}^{2}$ of holes in the plane if there is a continuous function $h:[0,1] \times[0,1] \rightarrow \mathbf{R}^{2}$ such that

(1) $h(0, t)=p(t)$ and $h(1, t)=q(t)$, for $t, 0 \leq$ $t \leq 1$

(2) $h(\lambda, 0)=p(0)=q(0)$ and $h(\lambda, 1)=p(1)=$ $q(1)$ for $0 \leq \lambda \leq 1$

(3) $h(\lambda, t) \notin F$ for $0 \leq \lambda \leq 1,0<t<1$

Let $T=\left\{w_{i}(0), w_{i}(1) ; 1 \leq i \leq l\right\}$ be the set of endpoints, also called terminals, of the paths in $W$ and let $F=T \cup O$. We call $F$ the set of features. A set $P=\left\{p_{1}, \ldots, p_{l}\right\}$ of paths is a homotopic shift of $W$ if

i.) $p_{i}$ is homotopic to $w_{i}$ with respect to $F, 1 \leq$ $i \leq l$ and

ii.) $P$ is collision-free.

For a set $S \subseteq \mathbf{R}^{2}$ let $U(S)=U_{1 / 2}(S)=\{x \in$ $\mathbf{R}^{2} ; \operatorname{dist}(x, s)<1 / 2$ for some $\left.s \in S\right\}$ be the open $1 / 2$ - neighborhood of $S$. For a path $p$ we frequently use $p$ to denote the set $\{p(t) ; 0 \leq t \leq 1\}$ of points of p. 
So a solution to a CHRP given by $O$ and $W$ consists of a homotopic shift $P$ of $W$ such that $U\left(p_{i}\right)$ is simply connected (simplicity condition), $U\left(p_{i}\right) \cap U(O)=\emptyset$ and $U\left(p_{i}\right) \cap U\left(p_{j}\right)=\emptyset \forall i$ and $j, i \neq j$ (disjointness condition). We call $P$ a $\lambda$ - solution if the above conditions hold for $U_{1 / 2 \cdot \lambda}$ instead of $U_{1 / 2}$.

A cut is either a straight-line segment connecting two features or a semi-infinite ray starting in a feature. In either case a cut must not cross any other feature. The capacity of a cut is its Euclidian length. For a cut $C$ and a path $w$ let $\operatorname{cr}(C, w)$ denote the number of points which $C$ and $w$ have in common, i.e.,

$$
\operatorname{cr}(C, w)=\mid\{t ; 0<t<1 \text { and } w(t) \in C\} \mid
$$

and let

$$
\operatorname{mincr}(C, w)=\min _{p \sim w} c r(C, p)
$$

be the minimal number of crossings of any homotopic shift of $w$ with $C$, cf. Figure 2. Note that crossings at the endpoints do not count. The density of a cut is given by

$$
\operatorname{dens}(C)=\sum_{w \in W} \operatorname{mincr}(C, w)
$$

We are now in a position to state our main theorem. For part b.) and c.) of the theorem we assume that the input paths are polygonal paths. We denote the number of bends in wire $w_{i}$ by $b_{i}$, the total number of bends by $b=\sum_{i} b_{i}$, the number of features by $m$ and the size of the input by $n=m+b$.

\section{Theorem 1:}

a.) A CHRP has a solution iff the cut condition holds, i.e., iff

$$
\operatorname{dens}(C)+1 \leq \operatorname{cap}(C)
$$

for all cuts $C$.

b.) The cut condition can be checked in time $O(b$. $\left.m+m^{2} \cdot \log b m\right)=O\left(n^{2} \cdot \log n\right)$.

c.) A solution (if there is one) can be constructed in time $O\left(b \cdot m^{2} \cdot \log b m\right)=O\left(n^{3} \cdot \log n\right)$ and space $O\left(\max _{i} b_{i} \cdot m^{2}\right)=O\left(n^{3}\right)$. Moreover, this solution minimizes the total path length.
The first results on homotopic one-layer routing are due to Cole/Siegel [CS] and Leiserson/Maley [LM]. They proved a result analoguous to theorem 1 for the $L_{\infty}$-Norm, i.e., dist corresponds to the Manhattan distance. Maley [M] extends the result to arbitrary polygonal distance functions and as a limiting case also to the Euclidian metric. His running time for part b.) is the same as ours and it is $O\left(n^{4} \cdot \log n\right)$ and space $O\left(n^{4}\right)$ for part c.). Moreover, he does not minimize the total path length. Our result was obtained independently of his (the papers [CS] and [LM] were the common basis) and the techniques used in parts a.) and c.) differ widely; part b.) is a simple generalization of [LM]. Mạley $[M]$ gives a very detailed proof for part a.) based on notions of combinatorial topology. His algorithm for part c.) is a slight generalization of [LM]. Our proof for part a.) is elementary and intuitive but may not fulfill the highest standards of rigour. Our main emphasis is on part c.) and we introduce a novel algorithmic idea there: sweeping the universal cover space.

Consider a fixed wire $w_{i}$ and let $s=w_{i}(0), t=w_{i}(1)$. The universal cover space of the plane with origin $s$ and with respect to the set $F$ of holes is given by $C=\left\{(x, p) ; x \in \mathbf{R}^{2}\right.$ and $p$ is the homotopy class of a path from $s$ to $x\}$;

cf. Figure 3, 4 and 5 for an illustration.

The solution path $p_{i}$ corresponding to $w_{i}$ is a shortest path from $(s, \epsilon)$ to $\left(t,\left[w_{i}\right]\right)$ in $\mathcal{C}-\mathcal{F}$ where $\mathcal{F}$ is the forbidden region for wire $w_{i}$, cf. section 3 and Figure 5. We construct the solution path $p_{i}$ for $w_{i}$ by a sweep of the cover space. More precisely, we first construct the rubberband equivalent $r b e_{0}\left(w_{i}\right)$, i.e., a shortest polygonal path homotopic to $w_{i}$. In Figure 1 the $w_{i}$ are already given by their rubberband equivalents (RBE). RBE's were introduced in [LM] and it was also shown how to construct them in [LM].

The path $r b e_{0}\left(w_{i}\right)$ is a path from $(s, \epsilon)$ to $\left(t,\left[w_{i}\right]\right)$ in $c$. Our idea is now to sweep a line perpendicular to 
$r b e_{0}\left(w_{i}\right)$ from $(s, \epsilon)$ to $\left(t,\left[w_{i}\right]\right)$ (at bends of $r b e_{0}\left(w_{i}\right)$ the line turns into a semi-infinite ray), and to construct the solution path $p_{i}$ as we move along, using the funnel method.

In the funnel method ([T]) we maintain a partition of the sweep line, where two points $x$ and $y$ belong to the same interval if the shortest paths to $x$ and $y$ are combinatorially the same and we maintain the current intersection of the sweep line with the forbidden region, cf. Figure 5.

The details of the algorithm can be found in section 3 ; section 2 contains a short discussion of part a.), and section 4 mentions some extensions.

It is natural to ask whether theorem 1 can be extended to vertex-disjoint routings in planar graphs. A partial answer is provided by theorem 2.

Let the problem shortest vertex-disjoint routings on a planar graph (with homotopies)

(SRPGH) be given by

a planar graph embedding $G$, vertex pairs $\left(s_{1}, t_{1}\right), \ldots$, $\left(s_{k}, t_{k}\right)$, a 'sketch' of the routings from $s_{i}$ to $t_{i}$ and a bound $b$ (integer).

Question: Do vertex-disjoint paths $s_{1} \rightarrow t_{1}, \ldots, s_{k} \rightarrow$ $t_{k}$ exist with the described homotopies of total length $\leq b$ ?

Theorem 2: SRPGH is NP - complete.

\section{Routability and the Cut Condition}

We briefly discuss the necessity and sufficiency of the cut condition. Let $P=\left\{p_{1}, \ldots, p_{l}\right\}$ be a solution to the CHRP and let $C$ be any cut connecting two features, say $f$ and $g$. Let $C^{0}=C-U(f)-U(g)$. It suffices to show that the length of $C^{0} \cap U\left(p_{i}\right)$ is at least $\operatorname{mincr}\left(C, w_{i}\right)$. Let $s, s^{\prime} \in\{f, g\} \cup \bigcup_{j}(C \cap$ $\left.p_{j}\right), s \neq s^{\prime}$. If $U(s) \cap U\left(s^{\prime}\right)=\emptyset$ for any such pair then we are done. So let us consider a pair $s, s^{\prime}$ with $U(s) \cap U\left(s^{\prime}\right) \neq \emptyset$. Note first that $s, s^{\prime} \in p_{i}$ for some $i$. This follows from the disjointness condition. Observe next that the subpath $p$ connecting $s$ and $s^{\prime}$ must be homotopic to the straight-line segment $\overline{s s^{\prime}}$ because of the simplicity condition. Thus we can remove the intersections $s$ and $s^{\prime}$ (or one of them if the other is a terminal) by a homotopic shift, cf. Figure 6. Continuing in this way we obtain a path $p_{i}^{\prime} \sim p_{i}$ such that $C \cap U\left(p_{i}^{\prime}\right) \subseteq C \cap U\left(p_{i}\right)$ and any two intersections of $p^{\prime}$ with $C$ have distance at least one. Thus the length of $C^{0} \cap U\left(p_{i}\right)$ is at least $\operatorname{mincr}\left(C, w_{i}\right)$ and the necessity of the cut condition is established.

For the proof of sufficiency and the algorithms we need some additional definitions. Let $p$ be a path and let $C$ be a cut. A path $p_{1}$ is an initial segment of $p$ if there is a continuous function $\alpha:[0,1] \rightarrow[0,1]$ such that $\alpha(t) \leq t$ and $p_{1}(t)=p(\alpha(t))$ for all $t$. A crossing of $p$ and $C$ is an initial segment $p_{1}$ of $p$ with $p_{1}(1) \in C$. Let $p$ and $q$ be paths with $p(1)=$ $q(0)$. Then the concatenation $p \circ q$ is defined by $(p \circ q)(t)=p(2 \cdot t)$ for $0 \leq t \leq 1 / 2$ and $(p \circ q)(t)=$ $q(2 \cdot t-1)$ for $1 / 2 \leq t \leq 1$.

Let $C$ be a cut and let $p$ and $q$ be paths with $p(0)=$ $q(0)$ and $p(1), q(1) \in C$. We call $p$ and $q C$-equivalent if $p$ is homotopic to $q \circ \overline{q(1) p(1)}$.

Let $C$ be a cut and $p_{1}$ a crossing of $p$ and $C$. Then the $C$-equivalence class of $p_{1}$ is called the type of the crossing $p_{1}$.

Let $C$ be a cut incident to a feature $f$, let $W=$ $\left\{w_{1}, \ldots, w_{l}\right\}$ be a given set of paths and let $h=$ $\sum_{i} \operatorname{mincr}\left(C, w_{i}\right)$.

We can shift $W$ into a collision-free set $P=\left\{p_{1}, \ldots, p_{l}\right\}$ with $h=\sum_{i} \operatorname{cr}\left(C, p_{i}\right)$ as follows. Let $p_{i}=w_{i}$ initially. If $\operatorname{mincr}\left(C, w_{i}\right)=\operatorname{cr}\left(C, p_{i}\right)$ for all $i$ then we are done. Otherwise there must be some $p_{j}$ such that two of the crossings of $p_{j}$ with $C$ can be removed by the operation shown in Figure 6. Proceeding in this fashion we obtain the desired set $P$ of paths.

Let $s_{1}, \ldots, s_{h}$ be the crossings of the paths in $P$ with the cut $C$ in the order of increasing distances from $f$, i.e., $\operatorname{dist}\left(f, s_{j}(1)\right)<\operatorname{dist}\left(f, s_{j+1}(1)\right)$ for $1 \leq j \leq$ $h-1$. Then $\left(\operatorname{type}\left(s_{1}\right), \ldots\right.$, type $\left.\left(s_{h}\right)\right)$ is called the sig- 
nature of $C$ with respect to $f$.

Note that the set $P$. defined above is not uniquely determined by $W$; nethertheless, the signature of $C$ is well-defined. Note also that if $\operatorname{mincr}\left(C, w_{i}\right)>0$ then some of the $s_{j}$ will be intersections of $p_{i}$ and $C$. We call the types of these $s_{j}$ the types of the required crossings of $w_{i}$ and $C$. Again, these types are independent of the particular set $P$ into which $W$ was shifted.

Let $\lambda$ be a positive real. A path $p$ is a $\lambda$-representative of a path $w$ if

- $p$ is homotopic to $w$ and if

- for all features $f$ and cuts $C$ incident to $f$ : if $p_{1}$ is a crossing of $p$ and $C$ and type $\left(p_{1}\right)=$ $t_{i}$, where $\left(t_{1}, t_{2}, \ldots\right)$ is the signature of $C$ with respect to $f$, then $\operatorname{dist}\left(f, p_{1}(1)\right) \geq \lambda \cdot i$.

A path $p$ is the $\lambda$-realization of $w$ if it is the $\lambda$ representative of shortest length. We denote the $\lambda$ realization by $r b e_{\lambda}(w)$.

Remark:

An alternative definition of $\lambda$-realization is as follows. Let $w$ be a path and let $s=w(0)$. Then the forbidden region for the $\lambda$-realization of $w$ is given by

$\mathcal{F}_{\lambda}=\{(y, p) \in \mathcal{C}$; there is a feature $f$, a cut $C$ incident to $f$ with signature $\left(t_{1}, t_{2}, \ldots\right)$, type $(q)=t_{i}$ is the type of a required crossing of $C$ and $w$ and $\operatorname{dist}(f, y)<\lambda \cdot i\}$.

Then the $\lambda$-realization of $w$ is the shortest path from $(s, \epsilon)$ to $(t,[w])$ in $C-F_{\lambda}$, cf. Figure $5 \mathrm{c}$.

Lemma 1: If the cut condition holds then the paths $r b e_{1}\left(w_{i}\right), 1 \leq i \leq l$, exist and form a solution to the CHRP.

Proof (sketch): Replace each wire $w_{i}$ by a rubberband of width $\lambda$ and replace each feature by a disk of diameter $\lambda$. Start with $\lambda=0$ and let $\lambda$ grow. A rubberband runs straight except if it is forced to run otherwise. In other words any bend of a path $r b e_{\lambda}\left(w_{i}\right)$ is forced. Suppose now that we cannot enlarge $\lambda$ all the way to $\lambda=1$. Then two rubberbands of opposite curvature must collide and hence the cut condition does not hold, cf. Figure 7.

q.e.d.

\section{Algorithms}

Throughout this section we will assume that our CHRP has a solution. Note that the cut condition can be checked in time $O\left(n^{2} \cdot \log n\right)$ by part b.) which is well below the target time for part $c$.).

The algorithm for part c.) is outlined in program 1.

1 Preprocessing (* time $O(b \cdot m \cdot \log b m) *$ )

2 for each $w \in W$ do

(* the loop body takes

$$
\text { time } \left.O\left(b_{w} \cdot m^{2} \cdot \log b m\right) *\right)
$$

2.1

for each $f \in F$ do

construct the forbidden region $\mathcal{F}(f)$ induced by $f$ od;

2.2

2.3

$$
\text { construct } \mathcal{F}=\bigcup_{f \in \mathcal{F}} \mathcal{F}(f)
$$$$
\text { construct } r b e_{1}(w)
$$

od;

\section{Program 1}

Let $w \in W, s=w(0), t=w(1)$. Let $C$ be the cover space with respect to origin $s$ and the set $F$ of holes: Let $\mathcal{F}=\mathcal{F}_{1}$ be the forbidden region as defined in the remark preceding lemma 1 . The solution path $r b e_{1}=r b e_{1}(w)$ is the shortest path from $(s, \epsilon)$ to $(t,[w])$ in $\mathcal{C}-\mathcal{F}$. We construct $r b e_{1}$ by application of the funnel method. The funnel method was introduced by Tompa $[\mathrm{T}]$ in the following situation, cf. Figure 8.

Given points $s$ and $t$ in the plane with the same $y$-coordinate and a set of obstacles, which are semiinfinite open vertical rays, construct a shortest path from $s$ to $t$. In the absence of obstacles the shortest path would be the horizontal line segment $\overline{s t}$. 
Tompa proposed to sweep a vertical line $(=$ a line perpendicular to the segment $\overline{s t}$ ) from $s$ to $t$ and to record for each intermediate position of the sweep line the current funnel; i.e., a partition of the sweep line into maximal intervals such that two points $x$ and $y$ belong to the same block iff the shortest paths from $s$ to $x$ and from $s$ to $y$ are combinatorially equivalent, i.e., bend at the same obstacles, cf. Figure 8.

The funnel changes whenever an obstacle is hit by the sweep line. It is either augmented by one additional interval (time $O(1)$ ) or it is reduced by one or more intervals (time $\mathrm{O}$ (number of discarded intervals)). Thus the running time of his method is proportional to the number of obstacles.

We extend the funnel method to our more general situation. There are two main difficulties:

i.) The obstacles have more complex shape, cf. Figure 5.

ii.) We do not sweep the plane but a more complex topological space.

Define the rubberband equivalent $r b e_{0}$ of the path $w$ to be the shortest path homotopic to $w$. (More precisely, $r b e_{0}$ is a shortest path in the closure of the set of paths homotopic to $w$. Note that $r b e_{0}$ goes through features and hence is not 'really' homotopic to $w$.) It is a polygonal path whose vertices are features. The rubberband equivalents of all wires can be constructed in time $O\left(n^{2} \cdot \log n\right)$, cf. [LM]. This constitutes the preprocessing phase.

For steps 2.1, 2.2 and 2.3 we use a sweep of the cover space which we now define. A connected subset $\mathcal{L} \subseteq \mathcal{C}$ is a straight-line (ray) if its projection $\operatorname{proj}(\mathcal{L})$ on the first coordinate is. A line $\mathcal{L}$ is perpendicular to $r b e_{0}$ at footpoint $(x, p) \in r b e_{0} \cap \mathcal{L}$ if $x$ lies in the interior of one of the straight-line segments constituting $r b e_{0}$ and if the projections are perpendicular. A ray $\mathcal{L}$ is perpendicular to $r b e_{0}$ at footpoint $(x, p)$ if $(x, p)$ is a vertex of $r b e_{0},(x, p)$ is the start point of ray $\mathcal{L}$ and if $\mathcal{L}$ is contained in the cone defined by the two rays starting at $(x, p)$ and being perpendicular to the line segments of $r b e_{0}$ incident to $x, p$ ); cf. Figure 9 .

The sweep is now given by the continuous motion of the footpoint of a line (ray) perpendicular to $r b e_{0}$ from $(s, \epsilon)$ to $(t,[w])$, more precisely, if the footpoint is on one of the straight-line segments then the footpoint moves continuously and if the footpoint is a vertex then the ray turns continuously. Thus the sweep alternates between straight-line and angular motion.

Next we will explain the three steps 2.1, 2.2 and 2.3 in more detail.

Step 2.1: For a feature $f$ let

$\mathcal{F}(f)=\{(y, q)$; there is a cut $C$ incident to $f, C$ has signature $\left(t_{1}, t_{2}, \ldots\right), t_{i}=\operatorname{type}(q), t_{i}$ is the type of a required crossing of $w$ with $C$ and $\operatorname{dist}(y, f)<i\}$

be the forbidden region for $r b e_{1}$ induced by feature $f$. We show how to construct $\mathcal{F}(f)$ in time $O\left(b_{w}\right.$. $m \cdot \log b m$ ) for each feature $f$.

A sector of $r b e_{0}$ with respect to $f$ is a maximal subpath of $r b e_{0}$ which is monotonic with respect to $f$ and completely visible from $f$, i.e., rays starting in $f$ intersect the subpath only once and do not pass through a feature before they intersect the subpath. The sectors of $r b e_{0}$ correspond in a natural way to angular sectors with tip $f$, cf. Figure 5 and 10. Within each angular sector the boundary of $\mathcal{f}(f)$ is a circular arc of constant radius.

Next we show how to compute the sectors and circular arcs mentioned above. We first determine for each feature $f$ the sorted order of the other features around $f$ and then define $2 \cdot m-2$ rays for each $f$, one for each feature $g$ and one for each interval between two features. Next we compute for each wire $w$ and feature $f$ the intersections between the rays starting in $f$ and $r b e_{0}(w)$ by plane sweep and sort the intersections for each ray and each $r b e_{0}(w)$. At this point we have determined the rank of the intersection on the ray for each intersection of a ray 
and a rubberband, cf. Figure 11 . This constitutes a global preprocessing step for step 2.1 and takes time $O\left(b \cdot m^{2} \cdot \log b m\right)$.

From now on we consider again a fixed $w$ and $f$. The sectors of $r b e_{0}=r b e_{0}(w)$ with respect to $f$ are now easily computed. We move along rbe $e_{0}$ and stop at each vertex and at each intersection with a ray starting in $f$. At each stop one decides in time $O(1)$ whether the current sector ends. Also, the radius of the circular arc is given by the rank of any intersection within the sector. The entire process takes time $O\left(b_{w} \cdot m\right)$ and yields the circular arcs bounding $\mathcal{F}(f)$. For each circular arc we know the radius and the sector of $r b e_{0}$ which it constrains.

Let us call a circular arc visible at a certain position of the sweep if the sweep line intersects the arc, cf. Figure 12. The positions where an arc $a$ is visible form an interval which we will to compute in the following. Also note that if an arc is visible at all then one of its endpoints is visible.

Let $r$ be the starting ray of the sector of arc $a$ and let $(x, p)$ be the intersection of $r$ with $r b e_{0}$; the symmetric procedure is applied to the terminating ray. We position ourselves in point $(x, p)$ and move towards $t$ if

- $\operatorname{dist}(x, f) \geq i$ and the angle between the oriented line segment $\overline{x f}$ and the $r b e_{0}$ is $\geq \frac{\pi}{2}$ or if

- $\operatorname{dist}(x, f)<i$ and the angle is $<\frac{\pi}{2}$

and towards $s$ otherwise; this is a rudimentary form of the cover space sweep. Let us assume that we move towards $t$ and that the second case occurs; the other cases are similar.

During our walk along $r b e_{0}$ we maintain the intersection of the sweep line with the ray or arc. We stop whenever the sweep line passes through a feature or when it passes through the endpoint of the arc. In the former case we check whether the feature is between the sweep line and the ray or arc and if so we stop the sweep because from now on the ray or arc will not be visible from the footpoint of the sweep line. In the latter case we replace the ray by the arc at the starting point of the arc and stop the sweep at the endpoint of the arc, cf. Figure 12. The time used for this process is proportional to the number of features passed. This assumes that we sort the features with respect to $r b e_{0}$ in time $O\left(b_{w} \cdot m \cdot \log b_{w} m\right)$ beforehand. The crucial observation is now that for any fixed intersection of a ray $r$ and $r b e_{0}$ we will pass through any feature at most once and hence the time spent for each of the $b_{w} \cdot m$ intersections is $O(m)$ or $O\left(b_{w} \cdot m^{2}\right)$ in total.

We have now computed for each arc its visibility interval and step 2.1 is completed.

\section{Step 2.2: The Forbidden Region $\mathcal{F}$}

Step 2.1 provides us with at most $b_{w} \cdot m^{2}$ arcs and their intervals of visibility. The goal of step 2.2 is to find for each position of the sweep line the most constraining visible arc, i.e., the intersection of the boundary of $\xi$ with the sweep line. In general there will be two intersections, one constraining $r b e_{1}$ from the left, the other from the right.

We solve this problem by a sweep along $r b e_{0}$ from $(s, \epsilon)$ to $(t,[w])$. The sweep line datastructure $D S$ contains the intersections of the sweep line with some of the currently visible arcs. We maintain the following

\section{Invariant:}

If a currently visible arc is not contained in $D S$ then it will never be the most constraining arc at a later position of the sweep line.

The invariant implies that the most constraining arcs are always contained in $D S$. Note also that $D S$ contains at most $O\left(\mathrm{~m}^{2}\right)$ arcs.

We stop the sweep at four kinds of events

i.) a starting point of a visibility interval

ii.) a terminating point of a visibility interval which is currently in $D S$

iii.) a change of motion, from straight-line to angular or vice-versa

iv.) an intersection of two arcs in $D S$. 
In order to find these events we maintain two queues. The queue $Q_{A}$ contains all events of kind i.), ii.) and iii.) and is easily precomputed in time $O\left(b_{w} \cdot m^{2}\right.$. $\log b m$ ) using the output of step 2.1. The queue $Q_{B}$ contains all positions which lie on the current segment of the sweep (i.e., either on the same straightline or the same angular segment as the current footpoint) at which an intersection of two arcs adjacent in $D S$ is met by the sweep line.

We now describe the actions performed at the four kinds of events. The actions for events of kind i.) or ii.) are obvious. For kind i.) we insert an arc into $D S$ and update $Q_{B}$ (one deletion and two insertions); for kind ii.) we delete an arc from $D S$ and update $Q_{B}$ (two deletions and one insertion). Thus an action of type i.) or ii.) takes time $O(\log m)$ and there are $O\left(b_{w} \cdot m^{2}\right)$ of them.

For events of kind iii.) we scan through $D S$, compute for each adjacent pair of arcs their intersection and check whether this intersection is met during the next segment of the sweep. If so, the appropriate position of the sweep line is added to $Q_{B}$. This takes time $O(\log m)$ per pair, there are $O\left(m^{2}\right)$ pairs and there are $O\left(b_{w}\right)$ events of kind iii.).

For events of kind iv.) the sweep line meets the intersection of two arcs, say $a$ and $b$, where $a$ is the more constraining arc immediately after the intersection. We delete the arc $b$ from $D S$ und update $Q_{B}$. This takes time $O(\log m)$ per event; also, there are only $O\left(b_{w} \cdot m^{2}\right)$ events of kind iv.) since each such event eliminates an arc.

\section{Lemma 2 (Looser Lemma):}

The action needed for an event of kind iv.) maintains the invariant.

Proof (sketch): Let $b$ be the arc deleted in an event of kind iv.), let $f$ be the center of the arc and let $S$ be the corresponding sector. Let $r$ be the terminating ray of the sector and let $H$ be the closed half-plane bounded by the line supporting $r$ and not containing $S$, cf. Figure 13. The arc $a$ intersects $b$ and is more constraining than $b$ immediately after the intersec- tion. Since the sector $S$ does neither contain any feature between $f$ and $b$ nor between $f$ and $r b e_{0}$ we conclude that the center $g$ of arc $a$ lies in $H$. If the visibility interval of $a$ does not end before the visibility interval of $b$ then we are done. Assume otherwise. The visibility interval of $a$ can end for two possible reasons. Either we sweep across a feature between $r b e_{0}$ and $a$ or the arc $a$ ends. In the former case the visibility interval of $b$ would also end and hence only the latter case can apply. Let $A$ be the endpoint of $a$. The arc $a$ ends in $A$ for either of two reasons. Either a monotonous sector of $r b e_{0}$ ends at the ray $\overrightarrow{g A}$ or there is a feature $h$ on the line segment $\overrightarrow{g A}$. Again the former case cannot arise because the visibility interval of $b$ has not ended yet. In the latter case consider the constraint generated by $h$.

Lemma 3 (Strength Lemma):

Let $g$ and $h$ be features, let $r$ be a ray starting in $g$ and let $h$ lie between $g$ and $x=r \cap r b e_{0}$ on $r$. Assume also that there is no other feature on the line segment $\overline{h x}$. Let $i$ be the radius of the arc generated by $g$ immediately to the left of $h$ and let $j$ be the radius of the arc generated by $h$ in the direction $r$, cf. Figure 14. Then $j+\operatorname{dist}(h, g) \geq i$.

Proof (sketch): Consider ray $r^{\prime}$ immediately to the left of $r$. Then rbe is the i-th path crossing $r^{\prime}$ if we count intersections starting in $g$. Only dist $(g, h)$ of these paths can cross the ray between $g$ and $h$ or end at $h$ by the cut condition. Hence $r b e_{0}$ is at least the $(i-\operatorname{dist}(g, h))$-th path when counting starts in h. This proves $j \geq i-\operatorname{dist}(g, h)$.

q.e.d.

The constraint generated by $h$ is at least as strong on the ray $\overrightarrow{g h}$ as the constraint generated by $g$. Also, $h \in H$. Consider a ray immediately to the right of $\overrightarrow{g h}$. On this ray either the constraint generated by $h$ or the one generated by $g$ is stronger. In the former case we continue the argument with $h$ instead of $g$ and in the latter case we continue the argument with g.

q.e.d. 
Step 2.3: The construction of $r b e_{1}$

As an output of step 2.2 we get two sorted lists containing the most constraining arcs bounding the wire to be routed from both sides. We compute the shortest path through the corresponding routing region by a combination of the funnel method and the sweep algorithm used in step 2.2. Like in $[\mathrm{T}]$ the sweep line is organized as a double ended queue of tangents to the constraining arcs seperating the intervals on the sweep line. We update this queue each time we reach the next arc in the two lists. The fact that we can give an unambiguous course for each wire rests on the

Lemma 4 (Monotonicity Lemma):

The path $r b e_{1}$ intersects the sweep line exactly once for each position of the sweep line.

Proof (sketch): Note first that rbe intersects the sweep line exactly once for each position of the sweep (recall that we are in cover space). Thus, $r b e_{1}$ intersects at least once since $r b e_{1}$ and $r b e_{0}$ are homotopic.

So we still have to show that there is no more than one intersection.

Assume otherwise and consider again the process of growing up $r b e_{\lambda}$ from $r b e_{0}$ to $r b e_{1}$ and let $\lambda$ be maximal such that $r b e_{\lambda}$ intersects no sweep line twice. Then the sweep line is tangent to two arcs of $r b e_{\lambda}$ that have different curvature, cf. Figure 15. So the cut between the features who caused these arcs is oversaturated and $\lambda$ cannot be enlarged to $\lambda=1$.

q.e.d.

\section{Extensions}

The algorithm can also deal with the following two generalizations.

i.) Obstacles are line segments instead of points.

ii.) The nets are multi-terminal nets. In this case each wire $w_{i}$ is a tree, the leaves being the terminals. The trees $w_{i}$ are pairwise disjoint, $\mathrm{cf}$. figure 16. The output trees must be homotopic to the input trees and must satisfy the simplicity and the disjointness condition. Our algorithm solves this problem, but, of course, it can no longer construct a minimum length solution.

\section{Conclusion}

This paper gives another illustration of the versatility of the sweep line paradigm. In particular, we show that it can be applied to more complex spaces than Euclidean spaces.

\section{References}

[CS] R. Cole and A. Siegel, "River Routing every which way, but loose," 24th Annual Symposium on Foundations of Computer Science (November 1983), pp. 112-121.

[LM] C. E. Leiserson and F. M. Maley, "Algorithms for Routing and Testing Routability of Planar VLSI Layouts", 17th Annual ACM Symposium on Theory of Computing (May 1985), pp. 69 78.

[M] F. M. Maley, "Single-Layer Wire Routing", Ph. D. Thesis, Massachusetts Institute of Technology, August 1987.

[ST] H. Seifert and W. Threlfall, "Lehrbuch der Topologie", Chelsea Publishing Company, 1947.

[T] M. Tompa, "An Optimal Solution to a WireRouting Problem", Journal of Computer and System Sciences 23, pp. 127-150. 


\section{Figures}
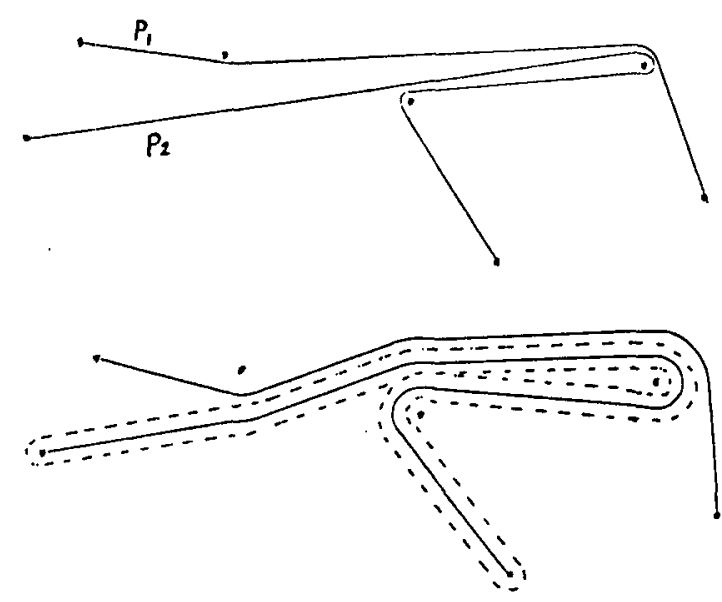

Figure 1: A CHRP and its solution. Obstacles and wire endpoints are shown as dots. $U\left(p_{2}\right)$ is indicated by a dashed line.

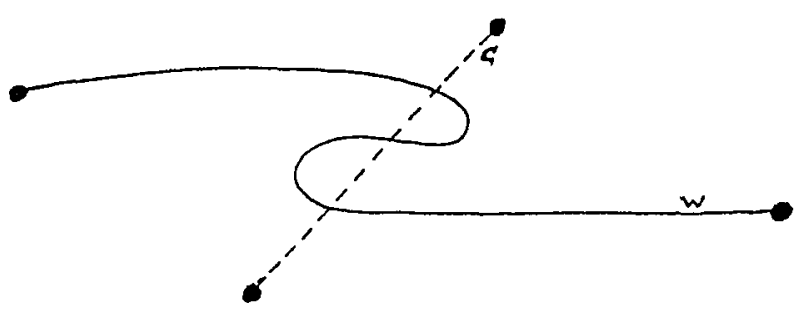

Figure 2: A cut $C$ is shown as a dashed line. The path $w$ has one necessary crossing with the cut, so $\operatorname{cr}(C, w)=3$ and $\operatorname{mincr}(C, w)=1$.

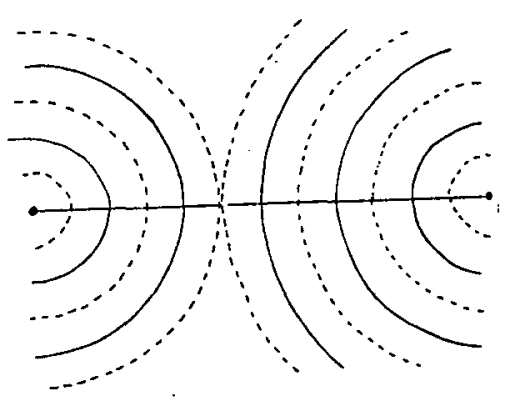

Figure 7: A collision of two rubberbands of opposite curvature gives rise to a cut whose density exceeds its capacity.
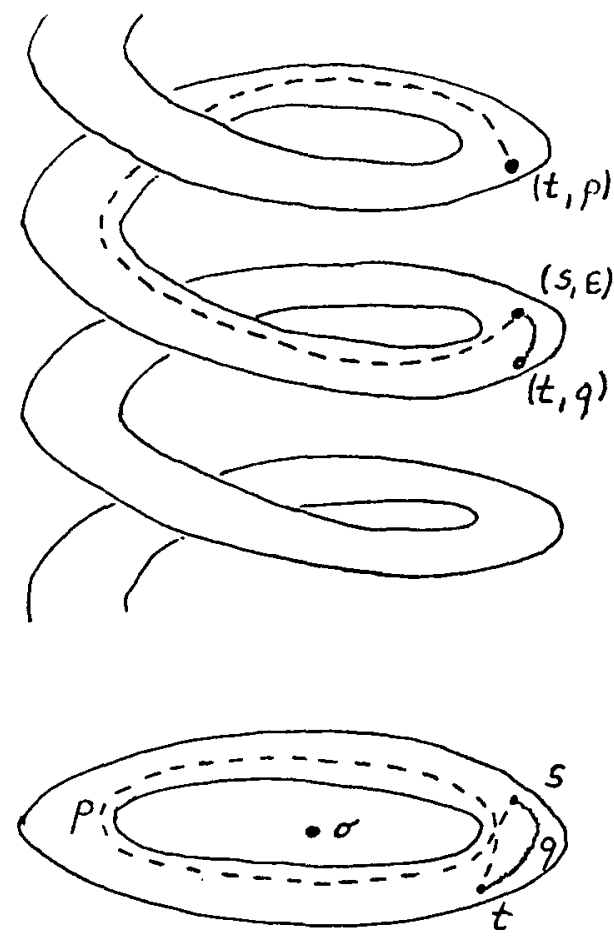

Figure 3: The bottom part shows the plane with a single obstacle $o$ and two paths $p$ and $q$ with terminals $s$ and $t$. The top part shows the universal cover space and the two paths in the cover space.

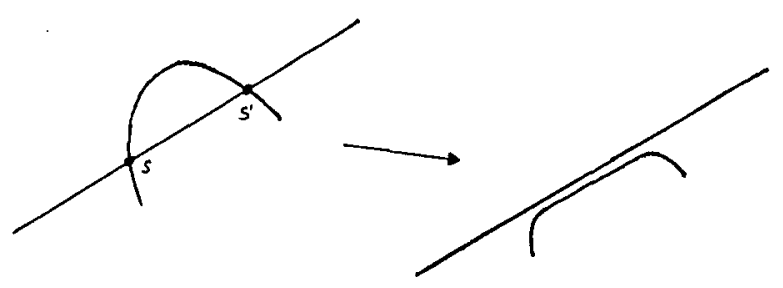

Figure 6: Removal of the two crossings $s$ and $s^{\prime}$.

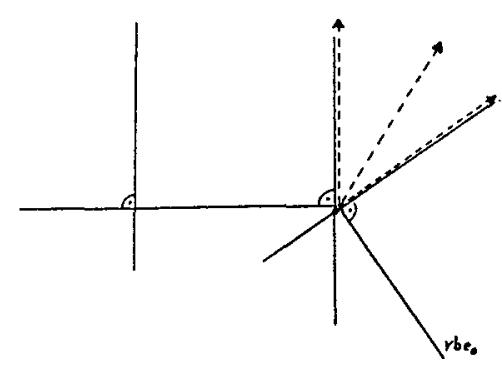

Figure 9: Lines (solid) and rays (dashed) perpendicular to $r b e_{0}$. 

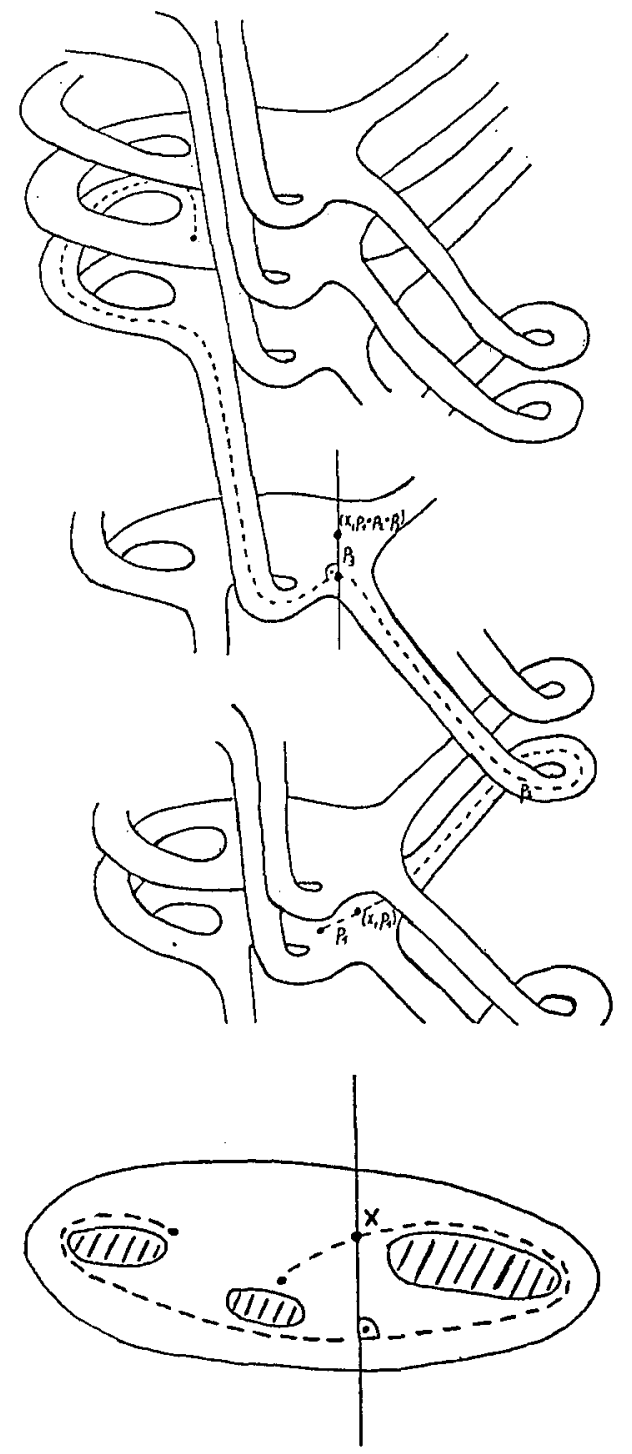

Figure 4: The bottom part shows the plane with three obstacles, a path and a position of the sweepline. The top part shows the situation in the cover space. The points $\left(x, p_{1}\right)$ and $\left(x, p_{1} \circ p_{2} \circ p_{3}\right)$ have the same projection into the plane.

5 a.)

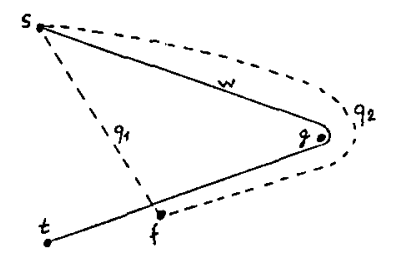

5 b.)

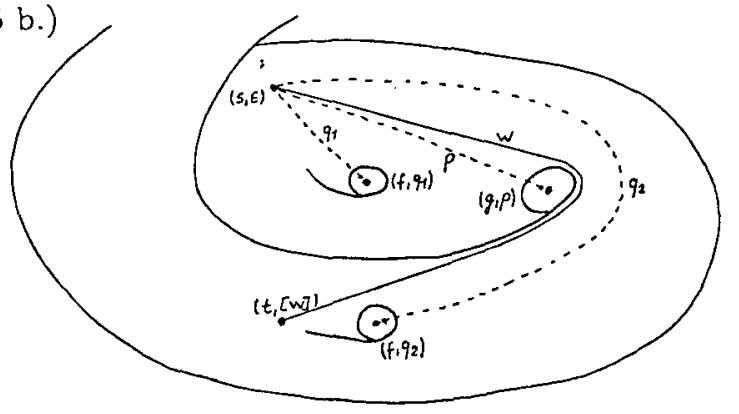

5 c.)

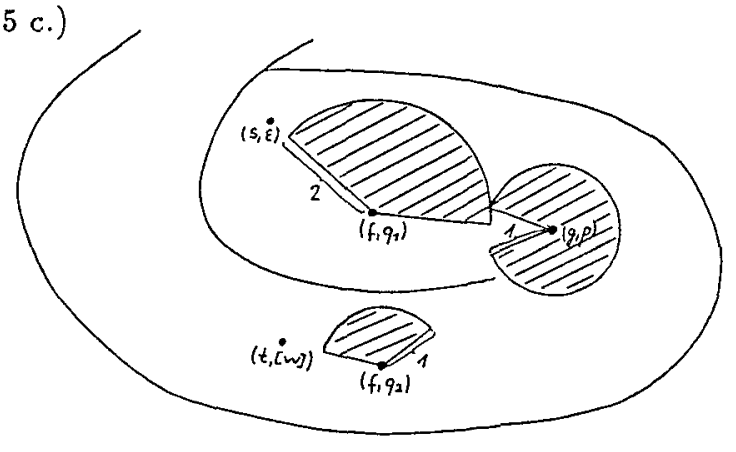

5 d.)

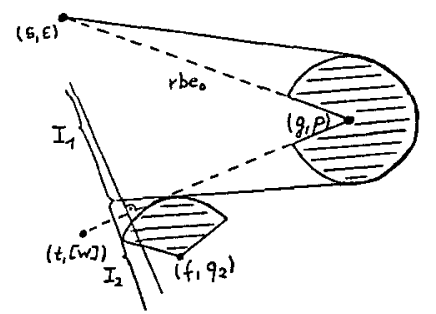

Figure 5: Part a.) shows a path $w$, two obstacles $f$ and $g$ and two paths $q_{1}$ and $q_{2}$ from $s$ to $f$. Part b.) shows the relevant part of the cover space. Observe the points $\left(f, q_{1}\right)$ and $\left(f, q_{2}\right)$ in the cover space. Part c.) shows the forbidden region $\mathcal{F}$. The forbidden region is the union of the three sectors, one with center $\left(f, q_{1}\right)$, radius 2 and rays $\overrightarrow{f s}$ and $\overrightarrow{f g}$, one with center $(g, p)$, radius 1 and rays $\overrightarrow{g s}$ and $\overrightarrow{g t}$, and one with center $\left(f, q_{2}\right)$, radius 1 and rays $\overrightarrow{f g}$ and $\overrightarrow{f t}$. Part d.) shows a position of the sweep-line. The RBE rbe $e_{0}$ is shown as a dashed line. The sweepline intersects the boundary of the sector with center $\left(f, q_{2}\right)$. The partition of the sweep-line consists of two intervals $I_{1}$ and $I_{2}$. For $x \in I_{1}$, the last segment on the path is a tangent to the obstacle with center $(g, p)$ and for $x \in I_{2}$ the last segment is a tangent to the obstacle with center $\left(f, q_{2}\right)$. 


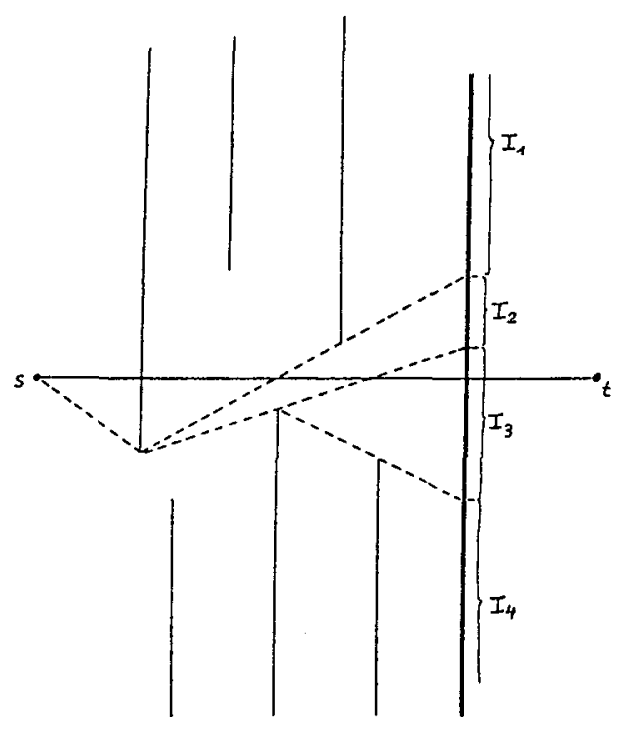

Figure 8: The funnel method ([T]). The current partition of the sweep-line consists of 4 intervals.

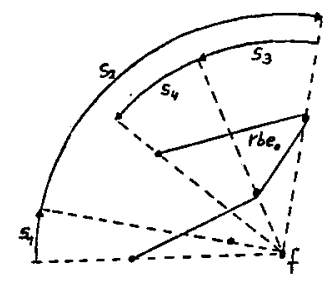

Figure 10: The sectors of an $r b e_{0}$ with respect to a feature $f$.

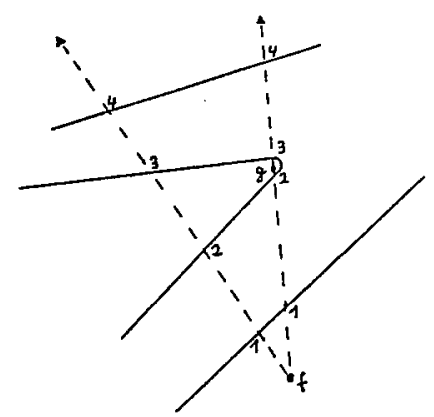

Figure 11: Two rays incident to a feature $f$ and the ranks of intersections between the rays and rubberbands.
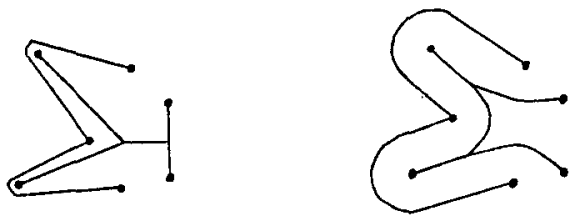

Figure 16: A multiterminal net problem and a solution for it.

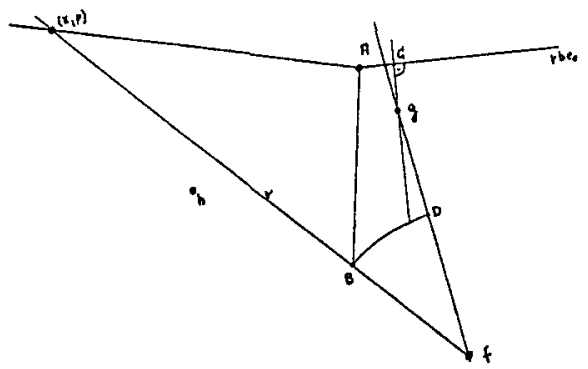

Figure 12: The arc $B D$ becomes visible when the sweep-line has footpoint $A$ and direction $\overrightarrow{A B}$ and stays visible until the footpoint is $C$. The intersection between the ray $r$ and $r b e_{0}$ is $(x, p)$. The ray stays visible when we sweep through $h$, the arc becomes invisible when we sweep through $g$.

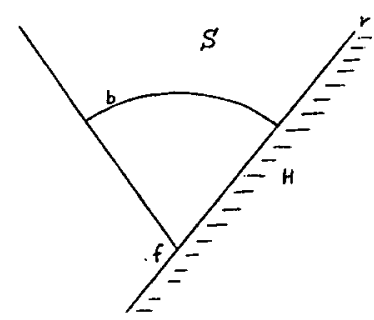

Figure 13

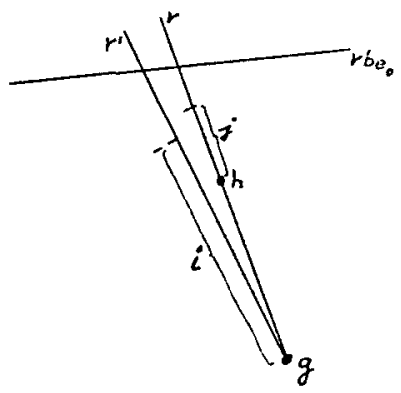

Figure 14

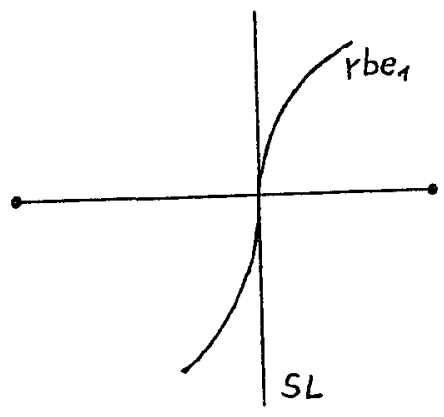

Figure 15 